\title{
THE EFFECT OF SPIN-ORBIT SCATTERING ON THE ELECTRICAL RESISTIVITY OF DISORDERED MATERIALS
}

\author{
B. Spisak and A. Paja \\ Department of Solid State Physics, Faculty of Physics and Nuclear Techniques \\ University of Mining and Metallurgy \\ Al. Mickiewicza 30, 30-059 Kraków, Poland
}

(Received April 26, 1999; revised version November 4, 1999)

The spin-orbit scattering of charge carriers is considered as an additional contribution to the potential scattering. The expressions for the effective cross-section and the relaxation time for disordered media are calculated. The results for the Coulomb screened potential are presented and compared with available experimental data.

PACS numbers: $71.70 . \mathrm{Ej}, 72.15 . \mathrm{Cz}$

\section{Introduction}

The theory of electron transport in disordered media such as liquid metals and amorphous alloys has been considerably developed since the fundamental papers by Ziman [1] and Faber and Ziman [2]. A very good review of the present knowledge in this field was given in the paper of Howson and Gallagher [3]. It is clear that the main contribution to the electrical resistivity in these materials comes from the elastic scattering of conduction electrons from a random array of potentials. There are some other mechanisms which can change this simple picture. One of them is the spin-orbit scattering which is to be discussed in this paper. It is important to take into account this contribution because it always exists and becomes significant in materials containing elements with higher atomic number $Z$. Many authors consider the inverse spin-orbit relaxation time $\tau_{\text {SO }}^{-1}$ which measures the strength of the spin-orbit coupling but they introduce $\tau_{\mathrm{SO}}^{-1}$ in a phenomenological way and estimate its value by fitting the theoretical formulae to the experimental curves for the resistivity or conductivity (see e.g. [4-7]). However, the modification of the inverse relaxation time $\tau^{-1}$ due to the spin-orbit scattering can be calculated from the quantum theory of scattering. 


\section{Model of the spin-orbit scattering}

Let us consider the scattering of the electron having spin $s=\hbar / 2$ off the array of the spherically symmetric atomic potentials $V_{\mathrm{a}}\left(\boldsymbol{r}-\boldsymbol{R}_{j}\right)$ located at random points $\boldsymbol{R}_{\boldsymbol{j}}$. The full potential which describes the spin-dependent scattering is

$$
V_{\mathrm{SO}}(r, \widehat{\sigma})=V(\boldsymbol{r})+a_{\mathrm{SO}} \widehat{\sigma} \cdot[\nabla V(r) \times p],
$$

where $V(r)=\sum_{j} V_{\mathrm{a}}\left(\boldsymbol{r}-\boldsymbol{R}_{j}\right), \widehat{\sigma}$ is the vector of the Pauli spin matrices, $\boldsymbol{p}=-\mathrm{i} \hbar \nabla$ is the momentum operator, $a_{\mathrm{SO}}=\frac{\hbar}{4 m^{2} c^{2}}$, and $\hbar, m, c$ have their usual meanings. The Schrödinger equation for the scattering of an electron from an ion has the form [8]:

$$
\left(\nabla^{2}+k^{2}\right) \Psi(r, \widehat{\sigma})=\frac{2 \mu}{\hbar^{2}} V_{\mathrm{SO}}(r, \widehat{\sigma}) \Psi(r, \widehat{\sigma}),
$$

where $\mu$ is the reduced mass of the electron and the ion, $\hbar^{2} k^{2} / 2 \mu$ is the energy of their relative movement and $\Psi(r, \widehat{\sigma})$ is a spinor

$$
\Psi(r, \widehat{\sigma})=\left(\begin{array}{l}
\psi_{1}(r) \\
\psi_{2}(r)
\end{array}\right)
$$

Its general solution for the initial state

$$
\Phi_{\mathrm{a}}(r, \widehat{\sigma})=\exp \left(\mathrm{i} k_{\mathrm{a}} \cdot r\right) \chi_{m_{S}}
$$

can be written as

$$
\Psi(r, \widehat{\sigma})=\Phi_{\mathrm{a}}(r, \widehat{\sigma})-\frac{\mu}{2 \pi \hbar^{2}} \int \mathrm{d}^{3} r^{\prime} \frac{\exp \left(\mathrm{i} k\left|r-r^{\prime}\right|\right)}{\left|r-r^{\prime}\right|} V_{\mathrm{SO}}\left(\boldsymbol{r}^{\prime}, \widehat{\sigma}\right) \Psi\left(\boldsymbol{r}^{\prime}, \widehat{\sigma}\right),
$$

where $\chi_{m_{s}}$ is a spin function

$$
\chi_{\frac{1}{2}}=\left(\begin{array}{l}
1 \\
0
\end{array}\right), \quad \chi_{-\frac{1}{2}}=\left(\begin{array}{l}
0 \\
1
\end{array}\right) .
$$

We replace the reduced mass $(\mu)$ by the electron mass $(m)$ henceforth because we consider the scattering of an electron from an ion at the position which is practically fixed.

The asymptotic form of the $\Psi(r, \widehat{\sigma})$ can be written as

$$
\Psi(r, \widehat{\sigma})=\Phi_{\mathrm{a}}(r, \widehat{\sigma})+F_{m_{S}} \frac{\exp (\mathrm{i} k r)}{r}
$$

where $F_{m_{S}}$ is the scattering amplitude. In the Born approximation we can write

$$
F_{m_{S}}=[A(\theta)+B(\theta)] \chi_{m_{S}},
$$

where

$$
\begin{aligned}
& A(\theta)=\frac{m}{2 \pi \hbar^{2}} \int \mathrm{d}^{3} r V(r) \exp \left[\mathrm{i}\left(k_{\mathrm{a}}-k_{\mathrm{b}}\right) \cdot r\right], \\
& B(\theta)=-\frac{m a_{\mathrm{SO}}}{2 \pi \hbar^{2}} \int \mathrm{d}^{3} r \exp \left(-\mathrm{i} k_{\mathrm{b}} \cdot r\right) \widehat{\sigma} \cdot[\nabla V(r) \times p] \exp \left(\mathrm{i} k_{\mathrm{a}} \cdot r\right) .
\end{aligned}
$$

The specific form of $F_{m_{S}}$ given by Eq. (8) resembles the fact that the $z$-component of the spin of the incident particle does not change due to the scattering from a spinless ion. Thus the theory presented here can be applied only for materials where the ions have closed electron shells after giving their outer electrons to the 
conduction band. The differential cross-section for unpolarized electrons is equal to the squared modulus of the scattering amplitude, averaged over two possible polarizations

$$
\frac{\mathrm{d} \sigma}{\mathrm{d} \Omega}=\frac{1}{2} \sum_{m_{S}}\left|F_{m_{S}}\right|^{2}=|A(\theta)|^{2}+\frac{1}{2} \sum_{m_{S}}\left|B(\theta) \chi_{m_{S}}\right|^{2} .
$$

The first term represents the usual potential scattering

$$
\frac{\mathrm{d} \sigma_{A}}{\mathrm{~d} \Omega}=\left|\frac{m}{2 \pi \hbar^{2}} \int \mathrm{d}^{3} r V(r) \exp (-\mathrm{i} \boldsymbol{q} \cdot r)\right|^{2},
$$

where $q$ gives the change of the wave vector due to scattering

$$
q=k_{\mathrm{b}}-k_{\mathrm{a}} \text {. }
$$

\section{The influence of the spin-orbit scattering on the relaxation times in disordered systems}

The effective cross-section is a configurational average of the expression (12). Using the definition of the structure factor for a monoatomic amorphous system

$$
S(\boldsymbol{q})=\frac{1}{N}\left\langle\sum_{j} \sum_{k} \exp \left[-\mathrm{i} \boldsymbol{q} \cdot\left(\boldsymbol{R}_{j}-\boldsymbol{R}_{k}\right)\right]\right\rangle,
$$

where the brackets denote a configurational averaging and $N$ is the number of atoms, after some straightforward calculations we can obtain [1]

$$
\frac{\mathrm{d} \sigma_{A}^{\text {eff }}}{\mathrm{d} \Omega}=\left(\frac{m}{2 \pi \hbar^{2}}\right) N S(\boldsymbol{q})\left|\widetilde{V}_{\mathrm{a}}(q)\right|^{2}
$$

where $\widetilde{V}_{\mathrm{a}}(q)$ is the Fourier transform of the atomic potential

$$
\widetilde{V}_{\mathrm{a}}(q)=\int \mathrm{d}^{3} r V_{\mathrm{a}}(r) \exp (-\mathrm{i} q \cdot r)
$$

and

$$
q=2 k \sin \frac{\theta}{2} .
$$

For the conduction electrons we assume $k$ equal to the Fermi vector $k_{\mathrm{F}}$. The second term in Eq. (11) represents the spin-orbit scattering. We have done an analogous calculation and obtained

$$
\frac{\mathrm{d} \sigma_{B}^{\mathrm{eff}}}{\mathrm{d} \Omega}=\frac{m^{2} a_{\mathrm{SO}}^{2}}{8 \pi^{2} \hbar^{2}} N S(q) \sum_{m_{S}}\left|\left[\left(\boldsymbol{U}_{\mathrm{a}}(q) \times k_{\mathrm{a}}\right) \cdot \hat{\sigma}\right] \chi_{m_{S}}\right|^{2},
$$

where $U_{\mathrm{a}}(q)$ is the Fourier transform of the gradient of $V_{\mathrm{a}}$ :

$$
U_{\mathrm{a}}(q)=\int \mathrm{d}^{3} r\left[\nabla V_{\mathrm{a}}(r)\right] \exp (-\mathrm{i} \boldsymbol{q} \cdot \boldsymbol{r}) .
$$

Now we can define the total transport relaxation time $\tau_{\text {tr }}$ :

$$
\frac{1}{\tau_{\mathrm{tr}}}=\frac{\hbar k_{\mathrm{F}}}{m} \frac{2 \pi}{V} \int_{0}^{\pi} \mathrm{d} \theta \sin \theta \frac{\mathrm{d} \sigma^{\text {eff }}}{\mathrm{d} \Omega}(1-\cos \theta)
$$

( $V$ is the volume of the sample), which allows us to calculate the resistivity $\rho$ :

$$
\rho=\frac{m}{n e^{2} \tau_{\mathrm{tr}}}
$$


where $n$ is the conduction electron concentration. Of course, $1 / \tau_{\text {tr }}$ is a sum of two contributions: $1 / \tau_{\mathrm{FZ}}$ and $1 / \tau_{\mathrm{SO}}$, coming from the usual Faber-Ziman treatment and from the spin-orbit scattering, respectively

$$
\frac{1}{\tau_{\mathrm{tr}}}=\frac{1}{\tau_{\mathrm{FZ}}}+\frac{1}{\tau_{\mathrm{SO}}}
$$

where

$$
\begin{aligned}
& \frac{1}{\tau_{\mathrm{FZ}}}=\frac{\hbar k_{\mathrm{F}}}{m} \frac{2 \pi}{V} \int_{0}^{\pi} \mathrm{d} \theta \sin \theta \frac{\mathrm{d} \sigma_{A}^{\mathrm{eff}}}{\mathrm{d} \Omega}(1-\cos \theta), \\
& \frac{1}{\tau_{\mathrm{SO}}}=\frac{\hbar k_{\mathrm{F}}}{m} \frac{2 \pi}{V} \int_{0}^{\pi} \mathrm{d} \theta \sin \theta \frac{\mathrm{d} \sigma_{B}^{\text {eff }}}{\mathrm{d} \Omega}(1-\cos \theta) .
\end{aligned}
$$

The formulae simplify very much when the scattering potential $V_{\mathrm{a}}(\boldsymbol{r})$ is spherically symmetric

$$
\begin{aligned}
& \tilde{V}_{\mathrm{a}}(q)=4 \pi \int_{0}^{\infty} \mathrm{d} r r^{2} j_{0}(q r) V_{\mathrm{a}}(r), \\
& \boldsymbol{U}_{\mathrm{a}}(q)=\left(0,0,-4 \pi \mathrm{i} \int_{0}^{\infty} \mathrm{d} r r^{2} j_{1}(q r) \frac{\mathrm{d} V_{\mathrm{a}}}{\mathrm{d} r}\right), \\
& \left(\boldsymbol{U}_{\mathrm{a}}(q) \times k_{\mathrm{a}}\right) \cdot \hat{\sigma}=-\mathrm{i} \hat{\sigma} \cdot n \frac{4 \pi k_{\mathrm{F}}{ }^{2} \sin \theta}{q} \int_{0}^{\infty} \mathrm{d} r r^{2} j_{1}(q r) \frac{\mathrm{d} V_{\mathrm{a}}}{\mathrm{d} r},
\end{aligned}
$$

where $j_{0}(q r), j_{1}(q r)$ are the spherical Bessel functions and $n$ is a unit vector, perpendicular to the scattering plane.

Using Eq. (19) and denotation

$$
I(q)=\int_{0}^{\infty} \mathrm{d} r r^{2} j_{1}(q r) \frac{\mathrm{d} V_{\mathrm{a}}}{\mathrm{d} r}
$$

we can write

$$
\begin{aligned}
& \frac{1}{\tau_{\mathrm{FZ}}}=\frac{m}{4 \pi \hbar^{3} k_{\mathrm{F}}^{3}} \frac{N}{V} \int_{0}^{2 k_{\mathrm{F}}} \mathrm{d} q q^{3} S(q)\left|\tilde{V}_{\mathrm{a}}(q)\right|^{2}, \\
& \frac{1}{\tau_{\mathrm{SO}}}=\frac{\pi m a_{\mathrm{SO}}^{2}}{\hbar k_{\mathrm{F}}^{3}} \frac{N}{V} \int_{0}^{2 k_{\mathrm{F}}} \mathrm{d} q q^{3}\left[\left(2 k_{\mathrm{F}}\right)^{2}-q^{2}\right] S(q)|I(q)|^{2} .
\end{aligned}
$$

The expressions (29) and (30) can easily be generalised for two-component liquid or amorphous alloys

$$
\frac{1}{\tau_{\mathrm{FZ}}}=\frac{m}{4 \pi \hbar^{3} k_{\mathrm{F}}^{3}} \frac{N}{V} \int_{0}^{2 k_{F}} \mathrm{~d} q q^{3} \sum_{\alpha} \sum_{\beta} \sqrt{c_{\alpha} c_{\beta}} S_{\alpha \beta}(q) \tilde{V}_{\alpha}(q) \tilde{V}_{\beta}^{*}(q)
$$

and 


$$
\begin{aligned}
\frac{1}{\tau_{\mathrm{SO}}} & =\frac{\pi m a_{\mathrm{SO}}^{2}}{\hbar k_{\mathrm{F}}^{3}} \frac{N}{V} \\
& \times \int_{0}^{2 k_{\mathrm{F}}} \mathrm{d} q q^{3}\left[\left(2 k_{\mathrm{F}}\right)^{2}-q^{2}\right] \sum_{\alpha} \sum_{\beta} \sqrt{c_{\alpha} c_{\beta}} S_{\alpha \beta}(q) I_{\alpha}(q) I_{\beta}^{*}(q),
\end{aligned}
$$

where $c_{\alpha}, c_{\beta}$ are the atomic concentrations of $\alpha$ and $\beta$ components. $S_{\alpha \beta}$ are the Ashcroft-Langreth partial structure factors [9]

$$
\begin{aligned}
& S_{\alpha \beta}(q)=\left(N_{\alpha} N_{\beta}\right)^{-1 / 2}\left\langle\sum_{j=1}^{N_{\alpha}} \sum_{k=1}^{N_{\beta}} \exp \left[-\mathrm{i} \boldsymbol{q} \cdot\left(\boldsymbol{R}_{\alpha j}-\boldsymbol{R}_{\beta k}\right)\right]\right\rangle \\
& \quad-\left(N_{\alpha} N_{\beta}\right)^{1 / 2} \delta_{Q, 0},
\end{aligned}
$$

where $N_{\alpha}$ and $N_{\beta}$ are the numbers of atoms of $\alpha$ - and $\beta$-type, $\boldsymbol{R}_{\alpha j}$ and $\boldsymbol{R}_{\beta k}$ are their positions and $\delta_{Q, 0}$ is the Kronecker symbol. The integrals $I_{\alpha}$ and $I_{\beta}$ of course depend on the potential of the specific ion, $V_{\alpha}$ or $V_{\beta}$

$$
I_{\gamma}(q)=\int_{0}^{\infty} \mathrm{d} r r^{2} j_{1}(q r) \frac{\mathrm{d} V_{\gamma}}{\mathrm{d} r} .
$$

As an example we consider the screened Coulomb potentials as the scattering centres in a monoatomic disordered sample

$$
V_{\mathrm{a}}(r)=\frac{V_{0} \exp (-\lambda r)}{r}
$$

where $V_{0}$ is a strength of the potential and $\lambda^{-1}$ is the Thomas-Fermi screening radius. Using the explicit expressions for spherical Bessel functions and the recurrence relation $[10]$

$$
\frac{\mathrm{d}}{\mathrm{d} x}\left[x^{n+1} j_{n}(x)\right]=x^{n+1} j_{n-1}(x),
$$

for calculation of the integrals (25) and (28) we obtain

$$
\begin{aligned}
& \tilde{V}_{\mathrm{a}}(q)=\frac{4 \pi V_{0}}{\lambda^{2}+q^{2}}, \\
& I(q)=-\frac{q V_{0}}{\lambda^{2}+q^{2}} .
\end{aligned}
$$

Thus the calculation of the relaxation times and the resistivity is straightforward provided the structure factor $S(\boldsymbol{q})$ is known.

\section{Numerical results}

We tried to evaluate the numerical values of the inverse relaxation times $\tau_{\mathrm{FZ}}^{-1}$ and $\tau_{\text {SO }}^{-1}$ for a simple model. We assumed the Coulomb screened potential in the form as above and approximated the structure factor $S(q)$ by a quadratic function

$$
S(q)=b q^{2}
$$

where $b$ is a coefficient of appropriate dimensionality $\left(\mathrm{m}^{2}\right)$. We took advantage 
of the fact that for liquid alkali metals $2 k_{\mathrm{F}}<k_{\mathrm{p}}$ [11], where $k_{\mathrm{p}}$ is the position of the first peak of $S(q)$, and in this region the shape of $S(q)$ can actually be approximated by a parabola (see e.g. [12] for liquid Rb). Using this approximation and defining the dimensionless parameter $l=\lambda / 2 k_{\mathrm{F}}$ we obtained

$$
\frac{1}{\tau_{\mathrm{FZ}}}=\frac{16 \pi m V_{0}^{2} b}{\hbar^{3} k_{\mathrm{F}}} \frac{N}{V} I_{\mathrm{FZ}}(l)
$$

where

$$
I_{\mathrm{FZ}}(l)=\int_{0}^{1} \mathrm{~d} x \frac{x^{5}}{\left(x^{2}+l^{2}\right)^{2}}
$$

and

$$
\frac{1}{\tau_{\mathrm{SO}}}=\frac{64 b m a_{\mathrm{SO}}^{2} V_{0}^{2} k_{\mathrm{F}}^{3}}{\hbar} \frac{N}{V} I_{\mathrm{SO}}(l)
$$

where

$$
I_{\mathrm{SO}}(l)=\int_{0}^{1} \mathrm{~d} x \frac{x^{7}\left(1-x^{2}\right)}{\left(x^{2}+l^{2}\right)^{2}} .
$$

Now we can calculate the ratio

$$
\frac{\tau_{\mathrm{SO}}^{-1}}{\tau_{\mathrm{FZ}}^{-1}}=4 a_{\mathrm{SO}}^{2} \hbar^{2} k_{\mathrm{F}}^{4} \frac{I_{\mathrm{SO}}}{I_{\mathrm{FZ}}} .
$$

This ratio is in fact a function of a dimensionless parameter $\left(r_{\mathrm{S}} / a_{0}\right)$, where $r_{\mathrm{S}}$ is the radius of the sphere of the volume occupied by one conduction electron and $a_{0}$ is the Bohr radius because we can write the parameter $l$ in the form

$$
l=0.4075\left(r_{\mathrm{S}} / a_{0}\right)^{1 / 2} \text {. }
$$

The values of $\left(r_{\mathrm{s}} / a_{0}\right)$ are well known [13] and we plotted the ratio (44) for the alkali metals in Fig. 1.

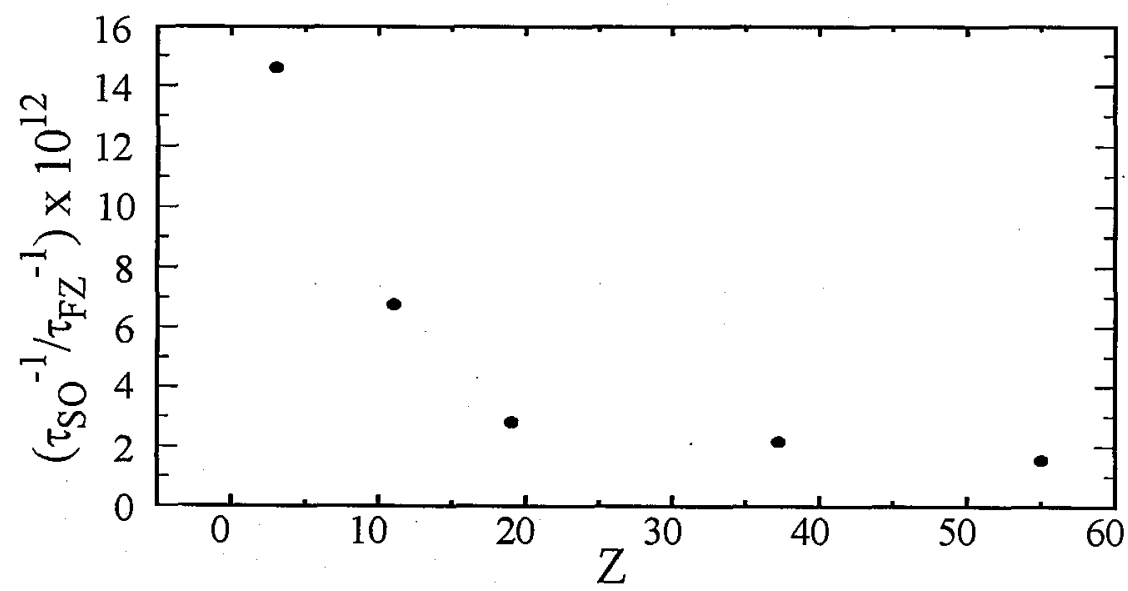

Fig. 1. The calculated ratio $\tau_{S O}^{-1} / \tau_{\mathrm{FZ}}^{-1}$ as a function of the atomic number $Z$ for five alkali metals: $\mathrm{Li}, \mathrm{Na}, \mathrm{K}, \mathrm{Rb}$, and $\mathrm{Cs}$. 
We can see that the ratio defined in Eq. (44) is of the order of $10^{-12}$. It means that the spin-orbit scattering gives very small contribution to the total scattering within this model. It is not surprising because the prefactor in the expression on the right hand side of Eq. (44) is of the order of $\left(v_{\mathrm{F}} / c\right)^{4} \approx 10^{-10}$, where $v_{\mathrm{F}}$ is the Fermi velocity. If we replaced the free electron mass $m$ in $a_{\text {SO }}$ by its effective mass $m^{*}$ then the ratio $\tau_{\mathrm{SO}}^{-1} / \tau_{\mathrm{FZ}}^{-1}$ would increase by the factor $m / m^{*}$, e.g. for $m^{*}=0.1 m$ we would obtain $\tau_{\mathrm{SO}}^{-1} / \tau_{\mathrm{FZ}}^{-1} \approx 10^{-8}$. It is more realistic approach because the charge carriers actually move through a disordered system of potentials, not in vacuum, but the discrepancies between the experimental data for the resistivity of alkali metals and the values obtained from the Faber-Ziman theory (see e.g. [14]) are still too big to be explained by this simple model. Perhaps the calculations based on the diffraction model (which is claimed to be good for liquid alkali metals) need to be improved first. Experimental data for amorphous alloys confirm our conclusion that $\tau_{\text {SO }}^{-1}$ is quite small though the results of various authors differ as to their numerical values. We give some examples in Table.

\section{TABLE}

The spin-orbit relaxation times $\tau_{\mathrm{SO}}$ (order of magnitude) for some amorphous materials and the ratio defined in Eq. (44). The Faber-Ziman relaxation time was assumed as $\tau_{\mathrm{FZ}}=10^{-16} \mathrm{~s}$, i.e. equal to the typical value of the elastic relaxation time.

\begin{tabular}{l|c|c|c}
\hline \hline Material & Reference & $\tau_{\text {SO }}[\mathrm{s}]$ & $\tau_{\mathrm{SO}}^{-1} / \tau_{\mathrm{FZ}}^{-1}$ \\
\hline $\mathrm{Ca}_{70} \mathrm{Al}_{30}$ & {$[4]$} & $10^{-11}$ & $10^{-5}$ \\
$\mathrm{Ca}_{60} \mathrm{Al}_{40}$ & {$[5]$} & $10^{-10}$ & $10^{-6}$ \\
$\mathrm{Ca}_{80} \mathrm{Al}_{20}$ & {$[5]$} & $10^{-10}$ & $10^{-6}$ \\
$\mathrm{Cu}_{65} \mathrm{Ti}_{35}$ & {$[6]$} & $10^{-12}$ & $10^{-4}$ \\
$\mathrm{Cu}_{36} \mathrm{Ti}_{64}$ & {$[7]$} & $10^{-11}$ & $10^{-5}$
\end{tabular}

We have to keep in mind that there is no direct method of measuring $\tau_{\mathrm{SO}}$ and everyone has to assume a specific theoretical model to estimate its value from experiment. Of course, the Faber-Ziman model in its primordial form cannot be applied for the materials listed in Table. The quoted authors estimated it from the weak localisation theories.

\section{Conclusions}

The contribution of the spin-orbit scattering to the total scattering of charge carriers in disordered media can be calculated within quantum theory of electron transport based on the Faber-Ziman diffraction model. We obtained general formulae in the first Born approximation for any position-dependent potential and analytical expressions for the Coulomb screened potential. The spin-orbit contribution to the resistivity calculated for liquid alkali metals turned out to be very 
small. It can be explained taking into account that the spin-orbit interaction actually is a relativistic effect. Thus the correction to the scattering potential is of the order of $\left(v_{F} / c\right)^{2}$ and to the effective cross-section is of the order of $\left(v_{\mathrm{F}} / c\right)^{4}$. The experimental data for amorphous alloys of metals other than alkali ones suggest that this contribution is essentially larger. Our work shows that more advanced quantum theory of spin-orbit scattering is needed to explain this enhancement.

\section{Acknowledgments}

The authors thank the Committee for Scientific Research for partial financial support.

\section{References}

[1] J.M. Ziman, Philos. Mag. 6, 1013 (1961).

[2] T.E. Faber, J.M. Ziman, Philos. Mag. 11, 153 (1965).

[3] M.A. Howson, B.L. Gallagher, Phys. Rep. 170, 269 (1988).

[4] A. Sahnoue, J.O. Strom-Olsen, H.E. Fischer, Phys. Rev. B 46, 10035 (1992).

[5] F.M. Mayea, M.A. Howson, J. Phys., Condens. Matter 4, 9355 (1992).

[6] P. Lindqvist, J. Phys., Condens. Matter 4, 177 (1992).

[7] A.K. Bhatnagar, G. Fritsch, D.G. Naugle, R. Haberkern, M. Kandlbinder, P. Lindqvist, Int. J. Mod. Phys. B 11, 303 (1997).

[8] A.S. Davydov, Quantum Mechanics, PWN, Warsaw 1967, p. 446 (in Polish).

[9] Y. Waseda, The Structure of Non-Crystalline Materials: Liquids and Amorphous Solids, McGraw Hill, New York 1980, p. 9.

[10] G.B. Arfken, H.J. Weber, Mathematical Methods for Physicists, 4th ed., Academic Press, San Diego 1995, p. 682.

[11] J.S. Dugdale, The Electrical Properties of Disordered Metals, Cambrige University Press, Cambridge 1995, p. 53.

[12] N.S. Gingrich, L. Heaton, J. Chem. Phys. 34, 873 (1961).

[13] N.W. Ashcroft, N.D. Mermin, Solid State Physics, PWN, Warsaw 1986, p. 25 (in Polish).

[14] T.E. Faber, in: The Physics of Metals, Ed. J.M. Ziman, Cambrige University Press, Cambridge 1969, p. 299. 